doi: $10.13108 / 2020-12-3-107$

\title{
SOLVABILITY OF HIGHER ORDER THREE-POINT ITERATIVE SYSTEMS
}

\section{K.R. PRASAD, M. RASHMITA, N. SREEDHAR}

\begin{abstract}
In this paper, we consider an iterative system of nonlinear $n^{\text {th }}$ order differential equations:

$$
y_{i}^{(n)}(t)+\lambda_{i} p_{i}(t) f_{i}\left(y_{i+1}(t)\right)=0, \quad 1 \leqslant i \leqslant m, \quad y_{m+1}(t)=y_{1}(t), \quad t \in[0,1],
$$
\end{abstract}

with three-point non-homogeneous boundary conditions

$$
\begin{gathered}
y_{i}(0)=y_{i}{ }^{\prime}(0)=\cdots=y_{i}^{(n-2)}(0)=0, \\
\alpha_{i} y_{i}^{(n-2)}(1)-\beta_{i} y_{i}^{(n-2)}(\eta)=\mu_{i}, \quad 1 \leqslant i \leqslant m,
\end{gathered}
$$

where $n \geqslant 3, \eta \in(0,1), \mu_{i} \in(0, \infty)$ is a parameter, $f_{i}: \mathbb{R}^{+} \rightarrow \mathbb{R}^{+}$is continuous, $p_{i}$ : $[0,1] \rightarrow \mathbb{R}^{+}$is continuous and $p_{i}$ does not vanish identically on any closed subinterval of $[0,1]$ for $1 \leqslant i \leqslant m$. We express the solution of the boundary value problem as a solution of an equivalent integral equation involving kernels and obtain bounds for these kernels. By an application of Guo-Krasnosel'skii fixed point theorem on a cone in a Banach space, we determine intervals of the eigenvalues $\lambda_{1}, \lambda_{2}, \cdots, \lambda_{m}$ for which the boundary value problem possesses a positive solution. As applications, we provide examples demonstrating our results.

Keywords: boundary value problem, iterative system, kernel, three-point, eigenvalues, cone, positive solution. Mathematics Subject Classification: 334B18, 34A40, 34B15

\section{INTRODUCTION}

The existence of positive solutions for multi-point boundary value problems associated with ordinary differential equations are of a high interest and play a vital role in different areas of applied mathematics and physics. Multi-point boundary value problems appear in the mathematical modelling of deflection of a curve beam having a constant or varying cross section, three layer beam, electromagnetic waves and so on. For example, the vibration of a guy wire of a uniform cross-section and composed of different parts with different densities can be formulated as multi-point boundary value problems.

Due to the importance in both theory and applications, much attention is focussed on obtaining optimal eigenvalue intervals for the existence of positive solutions of the iterative systems of nonlinear multi-point boundary value problems by an application of Guo-Krasnosel'skii fixed point theorem. A few papers along these lines are Henderson and Ntouyas [5], Henderson, Ntouyas and Purnaras [6, 7] and Prasad, Sreedhar and Kumar [14]. In the past, the researchers have focussed and established the existence of positive solutions of the boundary value problems associated with homogeneous boundary conditions,

K.R. Prasad, M. Rashmita, N. Sreedhar, Solvability of Higher order three-Point iterative SYSTEMS.

(C) Prasad K.R., Rashmita M., Sreedhar N. 2020.

Поступила 12 декабря 2019 г.

M. Rashmita is thankful to DST-INSPIRE, Government of India, New Delhi for awarding JRF. 
see [3, 13, 2, 12, 9, 18. However, some works have been carried out in establishing the existence of positive solutions of the boundary value problems with non-homogeneous boundary conditions, see [15, 17, 11, 16, 10].

Motivated by the papers mentioned above, in this paper, we determine intervals of the eigenvalues $\lambda_{1}, \lambda_{2}, \cdots, \lambda_{m}$, which will give guarantee for the existence of positive solutions of the iterative system of nonlinear $n^{\text {th }}$ order differential equations

$$
y_{i}^{(n)}(t)+\lambda_{i} p_{i}(t) f_{i}\left(y_{i+1}(t)\right)=0, \quad 1 \leqslant i \leqslant m, \quad y_{m+1}(t)=y_{1}(t), \quad t \in[0,1],
$$

satisfying three-point non-homogeneous boundary conditions

$$
y_{i}(0)=y_{i}^{\prime}(0)=\cdots=y_{i}^{(n-2)}(0)=0, \quad \alpha_{i} y_{i}^{(n-2)}(1)-\beta_{i} y_{i}^{(n-2)}(\eta)=\mu_{i}, \quad 1 \leqslant i \leqslant m,
$$

where $n \geqslant 3, \eta \in(0,1)$ and $\mu_{i} \in(0, \infty)$ is a parameter for $1 \leqslant i \leqslant m$. Our approach is based on application of Guo-Krasnosel'skii fixed point theorem on a cone in a Banach space.

Throughout the paper, we assume that the following conditions hold true:

(B1) $f_{i}: R^{+} \rightarrow R^{+}$is continuous for $1 \leqslant i \leqslant m$,

$(B 2) p_{i}:[0,1] \rightarrow R^{+}$is continuous and $p_{i}$ does not vanish identically on any closed subinterval of $[0,1]$ for $1 \leqslant i \leqslant m$,

(B3) $\alpha_{i}$ and $\beta_{i}$ are constants such that $\alpha_{i}>0$ and $\beta_{i} \in\left(0, \frac{\alpha_{i}}{\eta}\right)$ for $1 \leqslant i \leqslant m$,

(B4) each of

$$
f_{i 0}=\lim _{x \rightarrow 0^{+}} \frac{f_{i}(x)}{x} \text { and } f_{i \infty}=\lim _{x \rightarrow \infty} \frac{f_{i}(x)}{x}
$$

for $1 \leqslant i \leqslant m$ exists as positive real number.

The rest of the paper is organized as follows. In Section 2, we express the solution of the boundary value problem (1.1)-(1.2) as a solution of an equivalent integral equation involving kernels and find bounds for the these kernels. In Section 3, we establish the criteria determining the eigenvalues, for which the boundary value problems (1.1)-(1.2) has at least one positive solution in a cone; this is done by using the Guo-Krasnosel'skiis fixed point theorem. In Section 4 , as an application, we provide some examples to illustrate our results.

\section{KERNELS AND BOUNDS}

In this section, we express the solution of the boundary value problem (1.1)-(1.2) into an equivalent integral equation involving kernels by determining integral equation of $y_{i}$ for $1 \leqslant$ $i \leqslant m$ and find bounds for the kernels, which will be needed to establish the main results.

Lemma 2.1. If $h(t) \in C\left([0,1], R^{+}\right)$, then the boundary value problem

$$
y_{i}^{(n)}(t)+h(t)=0, \quad 1 \leqslant i \leqslant m, \quad t \in[0,1],
$$

with (1.2) has a unique solution and is given by

$$
y_{i}(t)=\frac{\mu_{i} t^{n-1}}{(n-1) !\left(\alpha_{i}-\eta \beta_{i}\right)}+\int_{0}^{1}\left[G(t, s)+\frac{\beta_{i} t^{n-1}}{(n-1) !\left(\alpha_{i}-\eta \beta_{i}\right)} G_{1}(\eta, s)\right] h(s) d s,
$$

where

$$
G(t, s)=\frac{1}{(n-1) !}\left\{\begin{array}{cl}
{\left[t^{n-1}(1-s)-(t-s)^{n-1}\right],} & 0 \leqslant s \leqslant t \leqslant 1 \\
t^{n-1}(1-s), & 0 \leqslant t \leqslant s \leqslant 1
\end{array}\right.
$$

and

$$
G_{1}(\eta, s)= \begin{cases}s(1-\eta), & 0 \leqslant s \leqslant \eta \leqslant 1 \\ \eta(1-s), & 0 \leqslant \eta \leqslant s \leqslant 1\end{cases}
$$


Proof. Let $y_{i}(t), 1 \leqslant i \leqslant m$, be the solution of boundary value problem (2.1), (1.2). Then an equivalent integral equation of (2.1) is given by

$$
y_{i}(t)=C_{0}+C_{1} t+C_{2} t^{2}+\cdots+C_{n-1} t^{n-1}-\frac{1}{(n-1) !} \int_{0}^{t}(t-s)^{n-1} h(s) d s .
$$

Using the boundary conditions $(1.2)$, we can determine $C_{j}$ as

$$
C_{j}=0 \quad \text { as } \quad j=0,1,2, \cdots, n-2
$$

and

$$
\begin{aligned}
C_{n-1}= & \frac{\mu_{i}}{(n-1) !\left(\alpha_{i}-\eta \beta_{i}\right)}+\frac{\alpha_{i}}{(n-1) !\left(\alpha_{i}-\eta \beta_{i}\right)} \int_{0}^{1}(1-s) h(s) d s \\
& -\frac{\beta_{i}}{(n-1) !\left(\alpha_{i}-\eta \beta_{i}\right)} \int_{0}^{\eta}(\eta-s) h(s) d s .
\end{aligned}
$$

Thus, the unique solution of boundary value problem (2.1), 1.2 is

$$
y_{i}(t)=\frac{\mu_{i} t^{n-1}}{(n-1) !\left(\alpha_{i}-\eta \beta_{i}\right)}+\int_{0}^{1}\left(G(t, s)+\frac{\beta_{i} t^{n-1}}{(n-1) !\left(\alpha_{i}-\eta \beta_{i}\right)} G_{1}(\eta, s)\right) h(s) d s .
$$

Lemma 2.2. Assume that the condition (B3) is satisfied. Then the kernels $G(t, s)$ and $G_{1}(t, s)$ are satisfies the following inequalities:

(i) $G(t, s) \geqslant 0$ and $G_{1}(t, s) \geqslant 0$ for all $t, s \in[0,1]$,

(ii) $G(t, s) \leqslant G(1, s)$ for all $t, s \in[0,1]$,

(iii) $G(t, s) \geqslant \frac{1}{4^{n-1}} G(1, s)$ for all $t \in I$ and $s \in[0,1]$, where $I=\left[\frac{1}{4}, \frac{3}{4}\right]$.

Proof. We prove the inequality $(i)$. For $0 \leqslant s \leqslant t \leqslant 1$, then we have

$$
\begin{aligned}
G(t, s) & =\frac{1}{(n-1) !}\left(t^{n-1}(1-s)-(t-s)^{n-1}\right) \geqslant \frac{1}{(n-1) !}\left(t^{n-1}(1-s)-(t-s t)^{n-1}\right) \\
& =\frac{t^{n-1}}{(n-1) !}\left((1-s)-(1-s)^{n-1}\right) \geqslant 0
\end{aligned}
$$

and

$$
G_{1}(t, s)=s(1-t) \geqslant 0 \text {. }
$$

For $0 \leqslant t \leqslant s \leqslant 1$, then we have

$$
G(t, s)=\frac{t^{n-1}(1-s)}{(n-1) !} \geqslant 0
$$

and

$$
G_{1}(t, s)=t(1-s) \geqslant 0 .
$$

Now, we prove the inequality $(i i)$. For $0 \leqslant s \leqslant t \leqslant 1$, then we have

$$
\frac{\partial}{\partial t} G(t, s)=\frac{1}{(n-2) !}\left(t^{n-2}(1-s)-(t-s)^{n-2}\right) \geqslant \frac{t^{n-2}}{(n-2) !}\left((1-s)-(1-s)^{n-2}\right) \geqslant 0 .
$$


For $0 \leqslant t \leqslant s \leqslant 1$, then we have

$$
\frac{\partial}{\partial t} G(t, s)=\frac{t^{n-2}}{(n-2) !}(1-s) \geqslant 0 .
$$

Therefore, $G(t, s)$ is increasing in $t$, which implies that $G(t, s) \leqslant G(1, s)$.

We proceed to proving inequality (iii). Hence, as $0 \leqslant s \leqslant t \leqslant 1$ and $t \in I$, we have

$$
\begin{aligned}
G(t, s) & =\frac{1}{(n-1) !}\left(t^{n-1}(1-s)-(t-s)^{n-1}\right) \\
& \geqslant \frac{t^{n-1}}{(n-1) !}\left((1-s)-(1-s)^{n-1}\right) \geqslant \frac{1}{4^{n-1}} G(1, s) .
\end{aligned}
$$

As $0 \leqslant t \leqslant s \leqslant 1$ and $t \in I$ we then get

$$
G(t, s)=\frac{t^{n-1}}{(n-1) !}(1-s) \geqslant \frac{1}{4^{n-1}} G(1, s) .
$$

We note that an $m$-tuple $\left(y_{1}(t), y_{2}(t), \cdots, y_{m}(t)\right)$ is a solution of the boundary value problem (1.1)-(1.2) if and only if $y_{i}(t)$ satisfies the following equations

$$
\begin{aligned}
y_{i}(t)= & \frac{\mu_{i} t^{n-1}}{(n-1) !\left(\alpha_{i}-\eta \beta_{i}\right)} \\
& +\lambda_{i} \int_{0}^{1}\left[G(t, s)+\frac{\beta_{i} t^{n-1}}{(n-1) !\left(\alpha_{i}-\eta \beta_{i}\right)} G_{1}(\eta, s)\right] p_{i}(s) f_{i}\left(y_{i+1}(s)\right) d s, \quad 1 \leqslant i \leqslant m, \quad t \in[0,1],
\end{aligned}
$$

and

$$
y_{m+1}(t)=y_{1}(t), \quad t \in[0,1]
$$

so that, in particular,

$$
\begin{aligned}
y_{1}(t) & =\frac{\mu_{1} t^{n-1}}{(n-1) !\left(\alpha_{1}-\eta \beta_{1}\right)}+\lambda_{1} \int_{0}^{1}\left(G\left(t, s_{1}\right)+\frac{\beta_{1} t^{n-1}}{(n-1) !\left(\alpha_{1}-\eta \beta_{1}\right)} G_{1}\left(\eta, s_{1}\right)\right) p_{1}\left(s_{1}\right) \\
& f_{1}\left(\frac{\mu_{2} s_{1}^{n-1}}{(n-1) !\left(\alpha_{2}-\eta \beta_{2}\right)}+\lambda_{2} \int_{0}^{1}\left(G\left(s_{1}, s_{2}\right)+\frac{\beta_{2} s_{1}^{n-1}}{(n-1) !\left(\alpha_{2}-\eta \beta_{2}\right)} G_{1}\left(\eta, s_{2}\right)\right) p_{2}\left(s_{2}\right)\right. \\
& \cdots f_{m-1}\left(\frac{\mu_{m} s_{m-1}^{n-1}}{(n-1) !\left(\alpha_{m}-\eta \beta_{m}\right)}+\lambda_{m} \int_{0}^{1}\left(G\left(s_{m-1}, s_{m}\right)+\frac{\beta_{m} s_{m-1}^{n-1}}{(n-1) !\left(\alpha_{m}-\eta \beta_{m}\right)} G_{1}\left(\eta, s_{m}\right)\right)\right. \\
& \left.\left.p_{m}\left(s_{m}\right) f_{m}\left(y_{1}\left(s_{m}\right)\right) d s_{m}\right) \cdots d s_{2}\right) d s_{1} .
\end{aligned}
$$

The following Guo-Krasnosel'skii fixed point theorem is a fundamental tool to establish our main results.

Theorem 2.1. [1, 4, 8] Let $B$ be a Banach space, $P \subseteq B$ be a cone and suppose that $\Omega_{1}, \Omega_{2}$ are open subsets of $B$ with $0 \in \Omega_{1}$ and $\bar{\Omega}_{1} \subset \Omega_{2}$. Suppose further that $T: P \cap\left(\bar{\Omega}_{2} \backslash \Omega_{1}\right) \rightarrow P$ is completely continuous operator such that either 
(i) $\|T y\| \leqslant\|y\|, y \in P \cap \partial \Omega_{1}$, and $\|T y\| \geqslant\|y\|, y \in P \cap \partial \Omega_{2}$,

or

(ii) $\|T y\| \geqslant\|y\|, y \in P \cap \partial \Omega_{1}$, and $\|T y\| \leqslant\|y\|, y \in P \cap \partial \Omega_{2}$.

Then $T$ has a fixed point in $P \cap\left(\bar{\Omega}_{2} \backslash \Omega_{1}\right)$.

\section{Positive SOlutions in A CONE}

In this section, we establish a criteria to determine the eigenvalues, for which the iterative system of three-point non-homogeneous boundary value problem $(1.1)-(1.2)$ has at least one positive solution in a cone.

Let $B=\{x: x \in C[0,1]\}$ be the Banach space equipped with the norm

$$
\|x\|=\max _{t \in[0,1]}|x(t)| .
$$

Define a cone $P \subset B$ by

$$
P=\left\{x \in B: x(t) \geqslant 0 \quad \text { on } \quad t \in[0,1] \quad \text { and } \quad \min _{t \in I} \quad x(t) \geqslant \frac{1}{4^{n-1}}\|x(t)\|\right\} .
$$

We define an operator $T: P \rightarrow B$ for $y_{1} \in P$ by

$$
\begin{aligned}
T y_{1}(t) & =\frac{\mu_{1} t^{n-1}}{(n-1) !\left(\alpha_{1}-\eta \beta_{1}\right)}+\lambda_{1} \int_{0}^{1}\left(G\left(t, s_{1}\right)+\frac{\beta_{1} t^{n-1}}{(n-1) !\left(\alpha_{1}-\eta \beta_{1}\right)} G_{1}\left(\eta, s_{1}\right)\right) p_{1}\left(s_{1}\right) \\
& f_{1}\left(\frac{\mu_{2} s_{1}^{n-1}}{(n-1) !\left(\alpha_{2}-\eta \beta_{2}\right)}+\lambda_{2} \int_{0}^{1}\left(G\left(s_{1}, s_{2}\right)+\frac{\beta_{2} s_{1}^{n-1}}{(n-1) !\left(\alpha_{2}-\eta \beta_{2}\right)} G_{1}\left(\eta, s_{2}\right)\right)\right. \\
& p_{2}\left(s_{2}\right) \cdots f_{m-1}\left(\frac{\mu_{m} s_{m-1}^{n-1}}{(n-1) !\left(\alpha_{m}-\eta \beta_{m}\right)}+\lambda_{m} \int_{0}^{1}\left(G\left(s_{m-1}, s_{m}\right)\right.\right. \\
& \left.\left.\left.+\frac{\beta_{m} s_{m-1}^{n-1}}{(n-1) !\left(\alpha_{m}-\eta \beta_{m}\right)} G_{1}\left(\eta, s_{m}\right)\right) p_{m}\left(s_{m}\right) f_{m}\left(y_{1}\left(s_{m}\right)\right) d s_{m}\right) \cdots d s_{2}\right) d s_{1} .
\end{aligned}
$$

Lemma 3.1. The operator $T: P \rightarrow B$ is a self map on $P$.

Proof. From the positivity of the kernels $G(t, s)$ and $G_{1}(t, s)$ in Lemma 2.2 that for $y_{1} \in P$, $T y_{1}(t) \geqslant 0$ on $t \in[0,1]$. Also, for $y_{1} \in P$, and by Lemma 2.2, we have

$$
\begin{aligned}
T y_{1}(t)= & \frac{\mu_{1} t^{n-1}}{(n-1) !\left(\alpha_{1}-\eta \beta_{1}\right)}+\lambda_{1} \int_{0}^{1}\left(G\left(t, s_{1}\right)+\frac{\beta_{1} t^{n-1}}{(n-1) !\left(\alpha_{1}-\eta \beta_{1}\right)} G_{1}\left(\eta, s_{1}\right)\right) \\
& p_{1}\left(s_{1}\right) f_{1}\left(\frac{\mu_{2} s_{1}^{n-1}}{(n-1) !\left(\alpha_{2}-\eta \beta_{2}\right)}+\lambda_{2} \int_{0}^{1}\left(G\left(s_{1}, s_{2}\right)+\frac{\beta_{2} s_{1}^{n-1}}{(n-1) !\left(\alpha_{2}-\eta \beta_{2}\right)}\right.\right. \\
& \left.G_{1}\left(\eta, s_{2}\right)\right) p_{2}\left(s_{2}\right) \cdots f_{m-1}\left(\frac{\mu_{m} s_{m-1}^{n-1}}{(n-1) !\left(\alpha_{m}-\eta \beta_{m}\right)}+\lambda_{m} \int_{0}^{1}\left(G\left(s_{m-1}, s_{m}\right)\right.\right. \\
& \left.\left.\left.+\frac{\beta_{m} s_{m-1}^{n-1}}{(n-1) !\left(\alpha_{m}-\eta \beta_{m}\right)} G_{1}\left(\eta, s_{m}\right)\right) p_{m}\left(s_{m}\right) f_{m}\left(y_{1}\left(s_{m}\right)\right) d s_{m}\right) \cdots d s_{2}\right) d s_{1}
\end{aligned}
$$




$$
\begin{aligned}
\leqslant & \frac{\mu_{1}}{(n-1) !\left(\alpha_{1}-\eta \beta_{1}\right)}+\lambda_{1} \int_{0}^{1}\left(G\left(1, s_{1}\right)+\frac{\beta_{1}}{(n-1) !\left(\alpha_{1}-\eta \beta_{1}\right)} G_{1}\left(\eta, s_{1}\right)\right) \\
& p_{1}\left(s_{1}\right) f_{1}\left(\frac{\mu_{2} s_{1}^{n-1}}{(n-1) !\left(\alpha_{2}-\eta \beta_{2}\right)}+\lambda_{2} \int_{0}^{1}\left(G\left(s_{1}, s_{2}\right)+\frac{\beta_{2} s_{1}^{n-1}}{(n-1) !\left(\alpha_{2}-\eta \beta_{2}\right)}\right.\right. \\
& \left.G_{1}\left(\eta, s_{2}\right)\right) p_{2}\left(s_{2}\right) \cdots f_{m-1}\left(\frac{\mu_{m} s_{m-1}^{n-1}}{(n-1) !\left(\alpha_{m}-\eta \beta_{m}\right)}+\lambda_{m} \int_{0}^{1}\left(G\left(s_{m-1}, s_{m}\right)\right.\right. \\
& \left.\left.\left.+\frac{\beta_{m} s_{m-1}^{n-1}}{(n-1) !\left(\alpha_{m}-\eta \beta_{m}\right)} G_{1}\left(\eta, s_{m}\right)\right) p_{m}\left(s_{m}\right) f_{m}\left(y_{1}\left(s_{m}\right)\right) d s_{m}\right) \cdots d s_{2}\right) d s_{1},
\end{aligned}
$$

so that,

$$
\begin{aligned}
\left\|T y_{1}(t)\right\| & \leqslant \frac{\mu_{1}}{(n-1) !\left(\alpha_{1}-\eta \beta_{1}\right)}+\lambda_{1} \int_{0}^{1}\left(G\left(1, s_{1}\right)+\frac{\beta_{1}}{(n-1) !\left(\alpha_{1}-\eta \beta_{1}\right)} G_{1}\left(\eta, s_{1}\right)\right) \\
& p_{1}\left(s_{1}\right) f_{1}\left(\frac{\mu_{2} s_{1}^{n-1}}{(n-1) !\left(\alpha_{2}-\eta \beta_{2}\right)}+\lambda_{2} \int_{0}^{1}\left(G\left(s_{1}, s_{2}\right)+\frac{\beta_{2} s_{1}^{n-1}}{(n-1) !\left(\alpha_{2}-\eta \beta_{2}\right)}\right.\right. \\
& \left.G_{1}\left(\eta, s_{2}\right)\right) p_{2}\left(s_{2}\right) \cdots f_{m-1}\left(\frac{\mu_{m} s_{m-1}^{n-1}}{(n-1) !\left(\alpha_{m}-\eta \beta_{m}\right)}+\lambda_{m} \int_{0}^{1}\left(G\left(s_{m-1}, s_{m}\right)\right.\right. \\
& \left.\left.\left.+\frac{\beta_{m} s_{m-1}^{n-1}}{(n-1) !\left(\alpha_{m}-\eta \beta_{m}\right)} G_{1}\left(\eta, s_{m}\right)\right) p_{m}\left(s_{m}\right) f_{m}\left(y_{1}\left(s_{m}\right)\right) d s_{m}\right) \cdots d s_{2}\right) d s_{1} .
\end{aligned}
$$

Further, if $y_{1} \in P$, we have from Lemma 2.2 and the above inequalities that

$$
\begin{aligned}
\min _{t \in I}\left(T y_{1}\right)(t)= & \min _{t \in I}\left\{\frac{\mu_{1} t^{n-1}}{(n-1) !\left(\alpha_{1}-\eta \beta_{1}\right)}+\lambda_{1} \int_{0}^{1}\left(G\left(t, s_{1}\right)+\frac{\beta_{1} t^{n-1}}{(n-1) !\left(\alpha_{1}-\eta \beta_{1}\right)} G_{1}\left(\eta, s_{1}\right)\right)\right. \\
& p_{1}\left(s_{1}\right) f_{1}\left(\frac{\mu_{2} s_{1}^{n-1}}{(n-1) !\left(\alpha_{2}-\eta \beta_{2}\right)}+\lambda_{2} \int_{0}^{1}\left(G\left(s_{1}, s_{2}\right)+\frac{\beta_{2} s_{1}^{n-1}}{(n-1) !\left(\alpha_{2}-\eta \beta_{2}\right)} G_{1}\left(\eta, s_{2}\right)\right)\right. \\
& p_{2}\left(s_{2}\right) \cdots f_{m-1}\left(\frac{\mu_{m} s_{m-1}^{n-1}}{(n-1) !\left(\alpha_{m}-\eta \beta_{m}\right)}+\lambda_{m} \int_{0}^{1}\left(G\left(s_{m-1}, s_{m}\right)+\right.\right. \\
& \left.\left.\left.\left.\frac{\beta_{m} s_{m-1}^{n-1}}{(n-1) !\left(\alpha_{m}-\eta \beta_{m}\right)} G_{1}\left(\eta, s_{m}\right)\right) p_{m}\left(s_{m}\right) f_{m}\left(y_{1}\left(s_{m}\right)\right) d s_{m}\right) \cdots d s_{2}\right) d s_{1}\right\} \\
\geqslant & \frac{1}{4^{n-1}}\left(\frac{\mu_{1}}{(n-1) !\left(\alpha_{1}-\eta \beta_{1}\right)}+\lambda_{1} \int_{0}^{1}\left(G\left(1, s_{1}\right)+\frac{\beta_{1}}{(n-1) !\left(\alpha_{1}-\eta \beta_{1}\right)} G_{1}\left(\eta, s_{1}\right)\right)\right. \\
& p_{1}\left(s_{1}\right) f_{1}\left(\frac{\mu_{2} s_{1}^{n-1}}{(n-1) !\left(\alpha_{2}-\eta \beta_{2}\right)}+\lambda_{2} \int_{0}^{1}\left(G\left(s_{1}, s_{2}\right)+\frac{\beta_{2} s_{1}^{n-1}}{(n-1) !\left(\alpha_{2}-\eta \beta_{2}\right)} G_{1}\left(\eta, s_{2}\right)\right)\right.
\end{aligned}
$$




$$
\begin{aligned}
& p_{2}\left(s_{2}\right) \cdots f_{m-1}\left(\frac{\mu_{m} s_{m-1}^{n-1}}{(n-1) !\left(\alpha_{m}-\eta \beta_{m}\right)}+\lambda_{m} \int_{0}^{1}\left(G\left(s_{m-1}, s_{m}\right)+\right.\right. \\
& \left.\left.\left.\left.\frac{\beta_{m} s_{m-1}^{n-1}}{(n-1) !\left(\alpha_{m}-\eta \beta_{m}\right)} G_{1}\left(\eta, s_{m}\right)\right) p_{m}\left(s_{m}\right) f_{m}\left(y_{1}\left(s_{m}\right)\right) d s_{m}\right) \cdots d s_{2}\right) d s_{1}\right) \\
\geqslant & \frac{1}{4^{n-1}}\left\|T y_{1}(t)\right\| .
\end{aligned}
$$

Therefore, $T: P \rightarrow P$ and this completes the proof.

Next, by an applications of the Arzela-Ascoli theorem, one can easily prove the operator $T$ is completely continuous.

Now, we seek suitable fixed point of the operator $T$ belonging to the cone $P$. To prove our result, we define positive numbers $F_{1}, F_{2}, F_{3}$ and $F_{4}$ by the formulae

$$
\begin{aligned}
& F_{1}=\max _{1 \leqslant i \leqslant m}\left\{\left(\frac{f_{i \infty}}{4^{2 n-2}} \int_{s \in I}\left(G(1, s)+\frac{\beta_{i}}{(n-1) !\left(\alpha_{i}-\eta \beta_{i}\right)} G_{1}(\eta, s)\right) p_{i}(s) d s\right)^{-1}\right\}, \\
& F_{2}=\min _{1 \leqslant i \leqslant m}\left\{\frac{1}{2}\left(f_{i 0} \int_{0}^{1}\left(G(1, s)+\frac{\beta_{i}}{(n-1) !\left(\alpha_{i}-\eta \beta_{i}\right)} G_{1}(\eta, s)\right) p_{i}(s) d s\right)^{-1}\right\}, \\
& F_{3}=\max _{1 \leqslant i \leqslant m}\left\{\left(\frac{f_{i 0}}{4^{2 n-2}} \int_{s \in I}\left(G(1, s)+\frac{\beta_{i}}{(n-1) !\left(\alpha_{i}-\eta \beta_{i}\right)} G_{1}(\eta, s)\right) p_{i}(s) d s\right)^{-1}\right\} \\
& F_{4}=\min _{1 \leqslant i \leqslant m}\left\{\frac{1}{2}\left(f_{i \infty} \int_{0}^{1}\left(G(1, s)+\frac{\beta_{i}}{(n-1) !\left(\alpha_{i}-\eta \beta_{i}\right)} G_{1}(\eta, s)\right) p_{i}(s) d s\right)^{-1}\right\} .
\end{aligned}
$$

Theorem 3.1. Assume that the conditions (B1)-(B4) are satisfied. Then, for each $\lambda_{1}, \lambda_{2}, \cdots, \lambda_{m}$ satisfying either

$$
F_{1}<\lambda_{1}<F_{2}, \quad F_{1}<\lambda_{2}<F_{2}, \cdots, F_{1}<\lambda_{m}<F_{2},
$$

or

$$
F_{3}<\lambda_{1}<F_{4}, \quad F_{3}<\lambda_{2}<F_{4}, \cdots, F_{3}<\lambda_{m}<F_{4},
$$

there exists an m-tuple $\left(y_{1}, y_{2}, \cdots, y_{m}\right)$ satisfying (1.1)-1.2) such that $y_{i}(t)>0$ on $(0,1)$ and $\mu_{i} \in(0, \infty)$ is sufficiently small for $1 \leqslant i \leqslant m$.

Proof. Let $\lambda_{i}, 1 \leqslant i \leqslant m$, be given as in (3.6). Let $\epsilon>0$ be chosen such that

$\max _{1 \leqslant i \leqslant m}\left\{\left(\frac{f_{i \infty}-\epsilon}{4^{2 n-2}} \int_{s \in I}\left[G(1, s)+\frac{\beta_{i}}{(n-1) !\left(\alpha_{i}-\eta \beta_{i}\right)} G_{1}(\eta, s)\right] p_{i}(s) d s\right)^{-1}\right\} \leqslant \min \left\{\lambda_{1}, \lambda_{2}, \cdots, \lambda_{m}\right\}$

and

$$
\begin{aligned}
\max & \left\{\lambda_{1}, \lambda_{2}, \cdots, \lambda_{m}\right\} \\
& \leqslant \min _{1 \leqslant i \leqslant m}\left\{\frac{1}{2}\left(\left(f_{i 0}+\epsilon\right) \int_{0}^{1}\left(G(1, s)+\frac{\beta_{i}}{(n-1) !\left(\alpha_{i}-\eta \beta_{i}\right)} G_{1}(\eta, s)\right) p_{i}(s) d s\right)^{-1}\right\} .
\end{aligned}
$$


Now, we seek a fixed point of the completely continuous operator $T: P \rightarrow P$ defined by (3.1). By the definition of $f_{i 0}, 1 \leqslant i \leqslant m$, there exists an $H_{1}>0$ such that, for each $1 \leqslant i \leqslant m$, the inequality

$$
f_{i}(x) \leqslant\left(f_{i 0}+\epsilon\right) x, \quad 0<x \leqslant H_{1},
$$

holds true.

Let $\mu_{i}, 1 \leqslant i \leqslant m$, be such that

$$
0<\mu_{i} \leqslant \frac{(n-1) !\left(\alpha_{i}-\eta \beta_{i}\right) H_{1}}{2} .
$$

Let $y_{1} \in P$ with $\left\|y_{1}\right\|=H_{1}$. By Lemma 2.2 and the choice of $\epsilon$, for $0 \leqslant s_{m-1} \leqslant 1$ we have

$$
\begin{aligned}
& \frac{\mu_{m} s_{m-1}^{n-1}}{(n-1) !\left(\alpha_{m}-\eta \beta_{m}\right)}+\lambda_{m} \int_{0}^{1}\left(G\left(s_{m-1}, s_{m}\right)+\frac{\beta_{m} s_{m-1}^{n-1}}{(n-1) !\left(\alpha_{m}-\eta \beta_{m}\right)} G_{1}\left(\eta, s_{m}\right)\right) \\
& \leqslant \frac{\mu_{m}\left(s_{m}\right) f_{m}\left(y_{1}\left(s_{m}\right)\right) d s_{m}}{(n-1) !\left(\alpha_{m}-\eta \beta_{m}\right)}+\lambda_{m} \int_{0}^{1}\left(G\left(1, s_{m}\right)+\frac{\beta_{m}}{(n-1) !\left(\alpha_{m}-\eta \beta_{m}\right)} G_{1}\left(\eta, s_{m}\right)\right) \\
& \leqslant \frac{H_{1}}{2}+\lambda_{m} \int_{0}^{1}\left(G\left(1, s_{m}\right)+\frac{\left.\beta_{m}\right)\left(f_{m 0}+\epsilon\right) y_{1}\left(s_{m}\right) d s_{m}}{(n-1) !\left(\alpha_{m}-\eta \beta_{m}\right)} G_{1}\left(\eta, s_{m}\right)\right) p_{m}\left(s_{m}\right) d s_{m}\left(f_{m 0}+\epsilon\right)\left\|y_{1}\right\| \\
& \leqslant \frac{H_{1}}{2}+\frac{H_{1}}{2}=H_{1} .
\end{aligned}
$$

In the same way, it follows from Lemma 2.2 and the choice of $\epsilon$ that, for $0 \leqslant s_{m-2} \leqslant 1$,

$$
\begin{aligned}
& \frac{\mu_{m-1} s_{m-2}^{n-1}}{(n-1) !\left(\alpha_{m-1}-\eta \beta_{m-1}\right)}+\lambda_{m-1} \int_{0}^{1}\left(G\left(s_{m-2}, s_{m-1}\right)+\frac{\beta_{m-1} s_{m-2}^{n-1}}{(n-1) !\left(\alpha_{m-1}-\eta \beta_{m-1}\right)}\right. \\
& \left.G_{1}\left(\eta, s_{m-1}\right)\right) p_{m-1}\left(s_{m-1}\right) f_{m-1}\left(\frac{\mu s_{m-1}^{n-1}}{(n-1) !\left(\alpha_{m}-\eta \beta_{m}\right)}+\lambda_{m} \int_{0}^{1}\left(G\left(s_{m-1}, s_{m}\right)\right.\right. \\
& \left.\left.+\frac{\beta_{m} s_{m-1}^{n-1}}{(n-1) !\left(\alpha_{m}-\eta \beta_{m}\right)} G_{1}\left(\eta, s_{m}\right)\right) p_{m}\left(s_{m}\right) f_{m}\left(y_{1}\left(s_{m}\right)\right) d s_{m}\right) d s_{m-1} \\
& \leqslant \frac{\mu_{m-1}}{(n-1) !\left(\alpha_{m-1}-\eta \beta_{m-1}\right)}+\lambda_{m-1} \int_{0}^{1}\left(G\left(1, s_{m-1}\right)+\frac{\beta_{m-1}}{(n-1) !\left(\alpha_{m-1}-\eta \beta_{m-1}\right)}\right. \\
& \left.G_{1}\left(\eta, s_{m-1}\right)\right) p_{m-1}\left(s_{m-1}\right) d s_{m-1}\left(f_{m-1,0}+\epsilon\right) H_{1} \\
& \leqslant \frac{H_{1}}{2}+\frac{H_{1}}{2}=H_{1} .
\end{aligned}
$$

Continuing with this bootstrapping argument, we have, for $0 \leqslant t \leqslant 1$,

$$
\frac{\mu_{1} t^{n-1}}{(n-1) !\left(\alpha_{1}-\eta \beta_{1}\right)}+\lambda_{1} \int_{0}^{1}\left(G\left(t, s_{1}\right)+\frac{\beta_{1} t^{n-1}}{(n-1) !\left(\alpha_{1}-\eta \beta_{1}\right)} G_{1}\left(\eta, s_{1}\right)\right) p_{1}\left(s_{1}\right)
$$




$$
\begin{aligned}
& f_{1}\left(\frac{\mu_{2} s_{1}^{n-1}}{(n-1) !\left(\alpha_{2}-\eta \beta_{2}\right)}+\lambda_{2} \int_{0}^{1}\left(G\left(s_{1}, s_{2}\right)+\frac{\beta_{2} s_{1}^{n-1}}{(n-1) !\left(\alpha_{2}-\eta \beta_{2}\right)} G_{1}\left(\eta, s_{2}\right)\right) p_{2}\left(s_{2}\right)\right. \\
& \cdots f_{m-1}\left(\frac{\mu_{m} s_{m-1}^{n-1}}{(n-1) !\left(\alpha_{m}-\eta \beta_{m}\right)}+\lambda_{m} \int_{0}^{1}\left(G\left(s_{m-1}, s_{m}\right)+\frac{\beta_{m} s_{m-1}^{n-1}}{(n-1) !\left(\alpha_{m}-\eta \beta_{m}\right)}\right.\right. \\
& \left.\left.\left.G_{1}\left(\eta, s_{m}\right)\right) p_{m}\left(s_{m}\right) f_{m}\left(y_{1}\left(s_{m}\right)\right) d s_{m}\right) \cdots d s_{2}\right) d s_{1} \leqslant H_{1},
\end{aligned}
$$

so that for $0 \leqslant t \leqslant 1$,

$$
T y_{1}(t) \leqslant H_{1}
$$

Hence, $\left\|T y_{1}\right\| \leqslant H_{1}=\left\|y_{1}\right\|$. If we let

$$
\Omega_{1}=\left\{x \in B:\|x\|<H_{1}\right\},
$$

then

$$
\left\|T y_{1}\right\| \leqslant\left\|y_{1}\right\| \quad \text { for } \quad y_{1} \in P \cap \partial \Omega_{1} \text {. }
$$

By the definitions of $f_{i \infty}, 1 \leqslant i \leqslant m$, there exists $\bar{H}_{2} \geqslant 0$, such that, for each $1 \leqslant i \leqslant m$,

$$
f_{i}(x) \geqslant\left(f_{i \infty}-\epsilon\right) x, \quad x \geqslant \bar{H}_{2} .
$$

Let

$$
H_{2}=\max \left\{2 H_{1}, 4^{n-1} \bar{H}_{2}\right\} .
$$

We choose $y_{1} \in P$ and $\left\|y_{1}\right\|=H_{2}$. Then

$$
\min _{t \in I} y_{1}(t) \geqslant \frac{1}{4^{n-1}}\left\|y_{1}\right\| \geqslant \bar{H}_{2} .
$$

By Lemma 2.2 and the choice of $\epsilon$, for $\frac{1}{4} \leqslant s_{m-1} \leqslant \frac{3}{4}$, we have:

$$
\begin{aligned}
& \frac{\mu_{m} s_{m-1}^{n-1}}{(n-1) !\left(\alpha_{m}-\eta \beta_{m}\right)}+\lambda_{m} \int_{0}^{1}\left(G\left(s_{m-1}, s_{m}\right)+\frac{\beta_{m} s_{m-1}^{n-1}}{(n-1) !\left(\alpha_{m}-\eta \beta_{m}\right)} G_{1}\left(\eta, s_{m}\right)\right) \\
& \geqslant \lambda_{m} \int_{0}^{1}\left(G\left(s_{m-1}, s_{m}\right)+\frac{\beta_{m}\left(s_{m}\right) f_{m}\left(y_{1}\left(s_{m}\right)\right) d s_{m}}{(n-1) !\left(\alpha_{m}-\eta \beta_{m}\right)} G_{1}\left(\eta, s_{m}\right)\right) p_{m}\left(s_{m}\right) f_{m}\left(y_{1}\left(s_{m}\right)\right) d s_{m} \\
& \geqslant \frac{1}{4^{n-1}} \lambda_{m} \int_{s \in I}\left(G\left(1, s_{m}\right)+\frac{\beta_{m}}{(n-1) !\left(\alpha_{m}-\eta \beta_{m}\right)} G_{1}\left(\eta, s_{m}\right)\right) p_{m}\left(s_{m}\right)\left(f_{m \infty}-\epsilon\right) y_{1}\left(s_{m}\right) d s_{m} \\
& \geqslant \frac{1}{4^{2 n-2}} \lambda_{m} \int_{s \in I}\left(G\left(1, s_{m}\right)+\frac{\beta_{m}}{(n-1) !\left(\alpha_{m}-\eta \beta_{m}\right)} G_{1}\left(\eta, s_{m}\right)\right) p_{m}\left(s_{m}\right) d s_{m}\left(f_{m \infty}-\epsilon\right)\left\|y_{1}\right\| \\
& \geqslant\left\|y_{1}\right\|=H_{2} .
\end{aligned}
$$

In the same way, it follows from Lemma 2.2 and the choice of $\epsilon$, that for $\frac{1}{4} \leqslant s_{m-1} \leqslant \frac{3}{4}$, we have

$$
\frac{\mu_{m-1} s_{m-2}^{n-1}}{(n-1) !\left(\alpha_{m-1}-\eta \beta_{m-1}\right)}+\lambda_{m-1} \int_{0}^{1}\left(G\left(s_{m-2}, s_{m-1}\right)+\frac{\beta_{m-1} s_{m-2}^{n-1}}{(n-1) !\left(\alpha_{m-1}-\eta \beta_{m-1}\right)}\right.
$$




$$
\begin{aligned}
& \left.G_{1}\left(\eta, s_{m-1}\right)\right) p_{m-1}\left(s_{m-1}\right) f_{m-1}\left(\frac{\mu_{m} s_{m-1}^{n-1}}{(n-1) !\left(\alpha_{m-1}-\eta \beta_{m-1}\right)}+\lambda_{m} \int_{0}^{1}\left(G\left(s_{m-1}, s_{m}\right)\right.\right. \\
& \left.\left.+\frac{\beta_{m-1} s_{m-1}^{n-1}}{(n-1) !\left(\alpha_{m-1}-\eta \beta_{m-1}\right)} G_{1}\left(\eta, s_{m}\right)\right) p_{m}\left(s_{m}\right) f_{m}\left(y_{1}\left(s_{m}\right)\right) d s_{m}\right) d s_{m-1} \\
\geqslant & \frac{1}{4^{n-1}} \lambda_{m-1} \int_{s \in I}\left(G\left(1, s_{m-1}\right)+\frac{\beta_{m-1}}{(n-1) !\left(\alpha_{m-1}-\eta \beta_{m-1}\right)} G_{1}\left(\eta, s_{m-1}\right)\right) \\
& p_{m-1}\left(s_{m-1}\right) d s_{m-1}\left(f_{m-1, \infty}-\epsilon\right) H_{2} \\
\geqslant & \frac{1}{4^{2 n-2}} \lambda_{m-1} \int_{s \in I}\left(G\left(1, s_{m-1}\right)+\frac{\beta_{m-1}}{(n-1) !\left(\alpha_{m-1}-\eta \beta_{m-1}\right)} G_{1}\left(\eta, s_{m-1}\right)\right) \\
& p_{m-1}\left(s_{m-1}\right) d s_{m-1}\left(f_{m-1, \infty}-\epsilon\right) H_{2} \geqslant H_{2} .
\end{aligned}
$$

Proceeding as above, we get:

$$
\begin{aligned}
& \frac{\mu_{1} t^{n-1}}{(n-1) !\left(\alpha_{1}-\eta \beta_{1}\right)}+\lambda_{1} \int_{0}^{1}\left(G\left(t, s_{1}\right)+\frac{\beta_{1} t^{n-1}}{(n-1) !\left(\alpha_{1}-\eta \beta_{1}\right)} G_{1}\left(\eta, s_{1}\right)\right) p_{1}\left(s_{1}\right) \\
& f_{1}\left(\frac{\mu_{2} s_{1}^{n-1}}{(n-1) !\left(\alpha_{2}-\eta \beta_{2}\right)}+\lambda_{2} \int_{0}^{1}\left(G\left(s_{1}, s_{2}\right)+\frac{\beta_{2} s_{1}^{n-1}}{(n-1) !\left(\alpha_{2}-\eta \beta_{2}\right)} G_{1}\left(\eta, s_{2}\right)\right) p_{2}\left(s_{2}\right)\right. \\
& \cdots f_{m-1}\left(\frac{\mu_{m} s_{m-1}^{n-1}}{(n-1) !\left(\alpha_{m-1}-\eta \beta_{m-1}\right)}+\lambda_{m} \int_{0}^{1}\left(G\left(s_{m-1}, s_{m}\right)+\frac{\beta_{m} s_{m-1}^{n-1}}{(n-1) !\left(\alpha_{m}-\eta \beta_{m}\right)}\right.\right. \\
& \left.\left.\left.G_{1}\left(\eta, s_{m}\right)\right) p_{m}\left(s_{m}\right) f_{m}\left(y_{1}\left(s_{m}\right)\right) d s_{m}\right) \cdots d s_{2}\right) d s_{1} \geqslant H_{2},
\end{aligned}
$$

so that, for $0 \leqslant t \leqslant 1$,

$$
T y_{1}(t) \geqslant H_{2}=\left\|y_{1}\right\|
$$

Hence, $\left\|T y_{1}\right\| \geqslant\left\|y_{1}\right\|$. If we let

$$
\Omega_{2}=\left\{x \in B:\|x\|<H_{2}\right\}
$$

then

$$
\left\|T y_{1}\right\| \geqslant\left\|y_{1}\right\|, \quad \text { for } \quad y_{1} \in P \cap \partial \Omega_{2} .
$$

Applying Theorem 2.1 to $(3.8)$ and $(3.9)$, we obtain that $T$ has a fixed point $y_{1} \in P \cap\left(\bar{\Omega}_{2} \backslash \Omega_{1}\right)$. Since $y_{m+1}=y_{1}$, we obtain a positive solution $\left(y_{1}, y_{2}, \cdots, y_{m}\right)$ of $1.1-(1.2)$ given iteratively by

$$
y_{i}(t)=\frac{\mu_{i} t^{n-1}}{(n-1) !\left(\alpha_{i}-\eta \beta_{i}\right)}+\lambda_{i} \int_{0}^{1}\left(G(t, s)+\frac{\beta_{i} t^{n-1}}{(n-1) !\left(\alpha_{i}-\eta \beta_{i}\right)} G_{1}(\eta, s)\right) p_{i}(s) f_{i}\left(y_{i+1}(s)\right) d s,
$$

as $i=m, m-1, \cdots, 1$.

Let $\lambda_{i}, 1 \leqslant i \leqslant m$, be given as in (3.7) and let $\epsilon>0$ be chosen such that

$$
\max _{1 \leqslant i \leqslant m}\left\{\left(\frac{f_{i 0}-\epsilon}{4^{2 n-2}} \int_{s \in I}\left(G(1, s)+\frac{\beta_{i}}{(n-1) !\left(\alpha_{i}-\eta \beta_{i}\right)} G_{1}(\eta, s)\right) p_{i}(s) d s\right)^{-1}\right\} \leqslant \min \left\{\lambda_{1}, \lambda_{2}, \cdots, \lambda_{m}\right\}
$$


and

$$
\begin{aligned}
& \max \left\{\lambda_{1}, \lambda_{2}, \cdots, \lambda_{m}\right\} \\
& \quad \leqslant \min _{1 \leqslant i \leqslant m}\left\{\frac{1}{2}\left(\int_{0}^{1}\left(G(1, s)+\frac{\beta_{i}}{(n-1) !\left(\alpha_{i}-\eta \beta_{i}\right)} G_{1}(\eta, s)\right) p_{i}(s) d s\left(f_{i \infty}+\epsilon\right)\right)^{-1}\right\} .
\end{aligned}
$$

We seek fixed point of the completely continuous operator $T: P \rightarrow P$ defined in (3.1). By the definition of $f_{i 0}, 1 \leqslant i \leqslant m$, there exists an $\bar{H}_{3}>0$ such that, for each $1 \leqslant i \leqslant m$,

$$
f_{i}(x) \geqslant\left(f_{i 0}-\epsilon\right) x, \quad 0<x \leqslant \bar{H}_{3} .
$$

Also, it follows from the definition of $f_{i 0}$ that $f_{i 0}=0,1 \leqslant i \leqslant m$, and so there exists $0<l_{m}<$ $l_{m-1}<\cdots<l_{2}<\bar{H}_{3}$ such that

$$
\begin{aligned}
\lambda_{i} f_{i}(x) & \leqslant \frac{l_{i-1}}{2 \int_{0}^{1}\left(G\left(1, s_{m}\right)+\frac{\beta_{i}}{(n-1) !\left(\alpha_{i}-\eta \beta_{i}\right)} G_{1}\left(\eta, s_{m}\right)\right) p_{i}(s) d s}, \quad x \in\left[0, l_{i}\right], \\
0 & <\mu_{i}<\frac{(n-1) !\left(\alpha_{i}-\eta \beta_{i}\right) l_{i-1}}{2} \text { for } 3 \leqslant i \leqslant m,
\end{aligned}
$$

and

$$
\begin{aligned}
\lambda_{2} f_{2}(x) & \leqslant \frac{\bar{H}_{3}}{2 \int_{0}^{1}\left(G\left(1, s_{m}\right)+\frac{\beta_{2}}{(n-1) !\left(\alpha_{2}-\eta \beta_{2}\right)} G_{1}\left(\eta, s_{m}\right)\right) p_{2}(s) d s}, \quad x \in\left[0, l_{2}\right], \\
0 & <\mu_{2}<\frac{(n-1) !\left(\alpha_{2}-\eta \beta_{2}\right) \bar{H}_{3}}{2} .
\end{aligned}
$$

Choose $y_{1} \in P$ with $\left\|y_{1}\right\|=l_{m}$. Then we have

$$
\begin{aligned}
& \frac{\mu_{m} s_{m-1}^{n-1}}{(n-1) !\left(\alpha_{m}-\eta \beta_{m}\right)}+\lambda_{m} \int_{0}^{1}\left(G\left(s_{m-1}, s_{m}\right)+\frac{\beta_{m} s_{m-1}^{n-1}}{(n-1) !\left(\alpha_{m}-\eta \beta_{m}\right)}\right. \\
& \left.G_{1}\left(\eta, s_{m}\right)\right) p_{m}\left(s_{m}\right) f_{m}\left(y_{1}\left(s_{m}\right)\right) d s_{m} \\
& \leqslant \frac{\mu_{m}}{(n-1) !\left(\alpha_{m}-\eta \beta_{m}\right)}+\lambda_{m} \int_{0}^{1}\left(G\left(1, s_{m}\right)+\frac{\beta_{m}}{(n-1) !\left(\alpha_{m}-\eta \beta_{m}\right)} G_{1}\left(\eta, s_{m}\right)\right) \\
& \leqslant \frac{l_{m-1}}{2}+\frac{p_{m}\left(s_{m}\right) f_{m}\left(y_{1}\left(s_{m}\right)\right) d s_{m}}{2 \int_{0}^{1}\left[G\left(1, s_{m}\right)+\frac{\beta_{m}}{(n-1) !\left(\alpha_{m}-\eta \beta_{m}\right)} G_{1}\left(\eta, s_{m}\right)\right] p_{m}\left(s_{m}\right) d s_{m}} \leqslant l_{m-1} .
\end{aligned}
$$

Continuing as above, we get:

$$
\begin{aligned}
& \frac{\mu_{2} s_{1}^{n-1}}{(n-1) !\left(\alpha_{2}-\eta \beta_{2}\right)}+\lambda_{2} \int_{0}^{1}\left(G\left(s_{1}, s_{2}\right)+\frac{\beta_{2} s_{1}^{n-1}}{(n-1) !\left(\alpha_{2}-\eta \beta_{2}\right)} G_{1}\left(\eta, s_{2}\right)\right) p_{2}\left(s_{2}\right) \\
& f_{2}\left(\frac{\mu_{3} s_{2}^{n-1}}{(n-1) !\left(\alpha_{3}-\eta \beta_{3}\right)}+\lambda_{3} \int_{0}^{1}\left(G\left(s_{2}, s_{3}\right)+\frac{\beta_{3} s_{2}^{n-1}}{(n-1) !\left(\alpha_{3}-\eta \beta_{3}\right)} G_{1}\left(\eta, s_{3}\right)\right)\right.
\end{aligned}
$$




$$
\left.p_{3}\left(s_{3}\right) \cdots f_{m}\left(y_{1}\left(s_{m}\right)\right) d s_{m} \cdots d s_{3}\right) d s_{2} \leqslant \bar{H}_{3} .
$$

Then,

$$
\begin{aligned}
T y_{1}(t) & =\frac{\mu_{1} t^{n-1}}{(n-1) !\left(\alpha_{1}-\eta \beta_{1}\right)}+\lambda_{1} \int_{0}^{1}\left(G\left(t, s_{1}\right)+\frac{\beta_{1} t^{n-1}}{(n-1) !\left(\alpha_{1}-\eta \beta_{1}\right)} G_{1}\left(\eta, s_{1}\right)\right) \\
& p_{1}\left(s_{1}\right) f_{1}\left(\frac{\mu_{2} s_{1}^{n-1}}{(n-1) !\left(\alpha_{2}-\eta \beta_{2}\right)}+\lambda_{2} \int_{0}^{1}\left(G\left(s_{1}, s_{2}\right)+\frac{\beta_{2} s_{1}^{n-1}}{(n-1) !\left(\alpha_{2}-\eta \beta_{2}\right)}\right.\right. \\
& \left.\left.G_{1}\left(\eta, s_{2}\right)\right) p_{2}\left(s_{2}\right) \cdots f_{m}\left(y_{1}\left(s_{m}\right)\right) d s_{m} \cdots d s_{2}\right) d s_{1} \\
\geqslant & \frac{\left(f_{10}-\epsilon\right)\left\|y_{1}\right\|}{4^{2 n-2}} \lambda_{1} \int_{s \in I}\left(G\left(1, s_{1}\right)+\frac{\beta_{1}}{(n-1) !\left(\alpha_{1}-\eta \beta_{1}\right)} G_{1}\left(\eta, s_{1}\right)\right) p_{1}\left(s_{1}\right) d s_{1} \\
\geqslant & \left\|y_{1}\right\| .
\end{aligned}
$$

So, $\left\|T y_{1}\right\| \geqslant\left\|y_{1}\right\|$. We let

$$
\Omega_{1}=\left\{x \in B:\|x\|<l_{m}\right\}
$$

then

$$
\left\|T y_{1}\right\| \geqslant\left\|y_{1}\right\| \quad \text { for } \quad y_{1} \in P \cap \partial \Omega_{1} .
$$

Since each $f_{i \infty}$ is assumed to be a positive real number, it follows that $f_{i}, 1 \leqslant i \leqslant m$, is unbounded at $\infty$. For each $1 \leqslant i \leqslant m$, let

$$
f_{i}^{*}(x)=\sup _{0 \leqslant s \leqslant x} f_{i}(s) .
$$

Then, it is straightforward that, for each $1 \leqslant i \leqslant m, f_{i}^{*}(x)$ is a non-decreasing real-valued function, $f_{i} \leqslant f_{i}^{*}$ and

$$
\lim _{x \rightarrow \infty} \frac{f_{i}^{*}(x)}{x}=f_{i \infty}
$$

By the definition of $f_{i \infty}, 1 \leqslant i \leqslant m$, there exists $\bar{H}_{4}$ such that, for each $1 \leqslant i \leqslant m$,

$$
f_{i}^{*}(x) \leqslant\left(f_{i \infty}+\epsilon\right) x, \quad x \geqslant \bar{H}_{4} .
$$

This implies that there exists $H_{4}>\max \left\{2 \bar{H}_{3}, \bar{H}_{4}\right\}$ such that, for each $1 \leqslant i \leqslant m$,

$$
f_{i}^{*}(x) \leqslant f_{i}^{*}\left(H_{4}\right), \quad 0<x \leqslant H_{4} .
$$

Let $\mu_{i}, 1 \leqslant i \leqslant m$, satisfy

$$
0<\mu_{i}<\frac{(n-1) !\left(\alpha_{i}-\eta \beta_{i}\right) H_{4}}{2} .
$$

We choose $y_{1} \in P$ with $\left\|y_{1}\right\|=H_{4}$. Then, using the above bootstrapping argument, we obtain

$$
\begin{gathered}
T y_{1}(t)=\frac{\mu_{1} t^{n-1}}{(n-1) !\left(\alpha_{1}-\eta \beta_{1}\right)}+\lambda_{1} \int_{0}^{1}\left(G\left(t, s_{1}\right)+\frac{\beta_{1} t^{n-1}}{(n-1) !\left(\alpha_{1}-\eta \beta_{1}\right)} G_{1}\left(\eta, s_{1}\right)\right) \\
p_{1}\left(s_{1}\right) f_{1}\left(\frac{\mu_{2} s_{1}^{n-1}}{(n-1) !\left(\alpha_{2}-\eta \beta_{2}\right)}+\lambda_{2} \int_{0}^{1}\left(G\left(s_{1}, s_{2}\right)+\frac{\beta_{2} s_{1}^{n-1}}{(n-1) !\left(\alpha_{2}-\eta \beta_{2}\right)}\right.\right.
\end{gathered}
$$




$$
\begin{aligned}
& \left.\left.G_{1}\left(\eta, s_{2}\right)\right) p_{2}\left(s_{2}\right) \cdots f_{m}\left(y_{1}\left(s_{m}\right)\right) d s_{m} \cdots d s_{2}\right) d s_{1} \\
\leqslant & \frac{\mu_{1}}{(n-1) !\left(\alpha_{1}-\eta \beta_{1}\right)}+\lambda_{1} \int_{0}^{1}\left(G\left(t, s_{1}\right)+\frac{\beta_{1}}{(n-1) !\left(\alpha_{1}-\eta \beta_{1}\right)} G_{1}\left(\eta, s_{1}\right)\right) \\
& p_{1}\left(s_{1}\right) f_{1}^{*}\left(\frac{\mu_{2} s_{1}^{n-1}}{(n-1) !\left(\alpha_{2}-\eta \beta_{2}\right)}+\lambda_{2} \int_{0}^{1}\left(G\left(s_{1}, s_{2}\right)+\frac{\beta_{2} s_{1}^{n-1}}{(n-1) !\left(\alpha_{2}-\eta \beta_{2}\right)}\right.\right. \\
& \left.\left.G_{1}\left(\eta, s_{2}\right)\right) p_{2}\left(s_{2}\right) \cdots f_{m}\left(y_{1}\left(s_{m}\right)\right) d s_{m} \cdots d s_{2}\right) d s_{1} \\
\leqslant & \frac{H_{4}}{2}+\lambda_{1} \int_{0}^{1}\left(G\left(1, s_{1}\right)+\frac{\beta_{1}}{(n-1) !\left(\alpha_{1}-\eta \beta_{1}\right)} G_{1}\left(\eta, s_{1}\right)\right) p_{1}\left(s_{1}\right) f_{1}^{*}\left(H_{4}\right) d s_{1} \\
\leqslant & \frac{H_{4}}{2}+\lambda_{1}\left(f_{1 \infty}+\epsilon\right) H_{4} \int_{0}^{1}\left(G\left(1, s_{1}\right)+\frac{\beta_{1}}{(n-1) !\left(\alpha_{1}-\eta \beta_{1}\right)} G_{1}\left(\eta, s_{1}\right)\right) p_{1}\left(s_{1}\right) d s_{1} \\
\leqslant & \frac{H_{4}}{2}+\frac{H_{4}}{2}=H_{4} \cdot
\end{aligned}
$$

Hence, $\left\|T y_{1}\right\| \leqslant\left\|y_{1}\right\|$. So, if we set

$$
\Omega_{2}=\left\{x \in B:\|x\|<H_{4}\right\}
$$

then

$$
\left\|T y_{1}\right\| \leqslant\left\|y_{1}\right\|, \quad \text { for } \quad y_{1} \in P \cap \partial \Omega_{2} .
$$

Applying Theorem 2.1 to $(3.10)$ and $(3.11)$, we obtain that $T$ has a fixed point $y_{1} \in P \cap\left(\bar{\Omega}_{2} \backslash \Omega_{1}\right)$. In view of the identity $y_{m+1}=y_{1}$ this yields that the $m$-tuple $\left(y_{1}, y_{2}, \cdots, y_{m}\right)$ satisfies boundary value problem $(1.1)-(1.2)$ for the values $\lambda_{i}, 1 \leqslant i \leqslant m$. The proof is complete.

\section{EXAMPLES}

Here we consider two examples demonstrating our results.

Example 1. Consider the iterative system of third order three-point non-homogeneous boundary value problem

$$
\begin{aligned}
& y_{1}^{\prime \prime \prime}+\lambda_{1} f_{1}\left(y_{2}(t)\right)=0, \quad t \in[0,1], \\
& y_{2}^{\prime \prime \prime}+\lambda_{2} f_{2}\left(y_{3}(t)\right)=0, \quad t \in[0,1], \\
& y_{3}^{\prime \prime \prime}+\lambda_{3} f_{3}\left(y_{1}(t)\right)=0, \quad t \in[0,1], \\
& y_{1}(0)=0, \quad y_{1}^{\prime}(0)=0, \quad 2 y_{1}^{\prime}(1)-3 y_{1}^{\prime}\left(\frac{1}{2}\right)=\mu_{1}, \\
& y_{2}(0)=0, \quad y_{2}^{\prime}(0)=0, \quad 3 y_{2}^{\prime}(1)-4 y_{2}^{\prime}\left(\frac{1}{2}\right)=\mu_{2}, \\
& y_{3}(0)=0, \quad y_{3}^{\prime}(0)=0, \quad 3 y_{3}^{\prime}(1)-2 y_{3}^{\prime}\left(\frac{1}{2}\right)=\mu_{3},
\end{aligned}
$$

where

$$
f_{1}\left(y_{2}\right)=y_{2}\left(476.5-468.7 e^{-y_{2}}\right)\left(210-202.7 e^{-3 y_{2}}\right),
$$




$$
\begin{aligned}
& f_{2}\left(y_{3}\right)=y_{3}\left(872.5-867.2 e^{-5 y_{3}}\right)\left(162-149.5 e^{-2 y_{3}}\right), \\
& f_{3}\left(y_{1}\right)=y_{1}\left(374.6-366.5 e^{-3 y_{1}}\right)\left(250-238.5 e^{-2 y_{1}}\right) .
\end{aligned}
$$

and

$$
p_{1}(t)=p_{2}(t)=p_{3}(t)=1 \text {. }
$$

The kernels $G(t, s)$ and $G_{1}(t, s)$ are

$$
G(t, s)=\frac{1}{2}\left\{\begin{array}{cc}
t^{2}(1-s)-(t-s)^{2}, & 0 \leqslant s \leqslant t \leqslant 1, \\
t^{2}(1-s), & 0 \leqslant t \leqslant s \leqslant 1,
\end{array}\right.
$$

and

$$
G_{1}\left(\frac{1}{2}, s\right)=\left\{\begin{array}{cl}
\frac{s}{2}, & 0 \leqslant s \leqslant \frac{1}{2} \leqslant 1, \\
\frac{(1-s)}{2}, & 0 \leqslant \frac{1}{2} \leqslant s \leqslant 1 .
\end{array}\right.
$$

By direct calculation, we find that

$$
\begin{aligned}
f_{10}= & 56.94, \quad f_{20}=66.25, \quad f_{30}=93.15 \\
f_{1 \infty}= & 100065, \quad f_{2 \infty}=141345, \quad f_{3 \infty}=93650 \\
F_{1}= & \max \left\{\left(\frac{1}{256} \int_{0.25}^{0.75}\left(G(1, s)+3 G_{1}\left(\frac{1}{2}, s\right)\right) d s(100065)\right)^{-1},\right. \\
& \left(\frac{1}{256} \int_{0.25}^{0.75}\left(G(1, s)+2 G_{1}\left(\frac{1}{2}, s\right)\right) d s(141345)\right)^{-1}, \\
& \left.\left(\frac{1}{256} \int_{0.25}^{0.75}\left(G(1, s)+(0.5) G_{1}\left(\frac{1}{2}, s\right)\right) d s(93650)\right)^{-1}\right\} \\
= & \max \{0.0031692,0.0032499,0.0149956\} \\
= & 0.0149956 \\
F_{2}= & \min \{0.019158895,0.022641543,0.0368069256\}=0.019158895 .
\end{aligned}
$$

Applying Theorem 3.1, we get an optimal eigenvalue interval $0.0149956<\lambda_{i}<0.019158895$, $i=1,2,3$ for which the boundary value problem 4.1 -4.2 has at least one positive solution by choosing $\mu_{1}, \mu_{2}$ and $\mu_{3}$ are sufficiently small.

Example 2. Here we consider the iterative system of third order three-point non-homogeneous boundary value problem

$$
\begin{array}{ll}
y_{1}^{\prime \prime \prime}+\lambda_{1} f_{1}\left(y_{2}(t)\right)=0, & t \in[0,1], \\
y_{2}^{\prime \prime \prime}+\lambda_{2} f_{2}\left(y_{3}(t)\right)=0, & t \in[0,1], \\
y_{3}^{\prime \prime \prime}+\lambda_{3} f_{3}\left(y_{1}(t)\right)=0, & t \in[0,1],
\end{array}
$$




$$
\begin{aligned}
& y_{1}(0)=0, \quad y_{1}^{\prime}(0)=0, \quad 2 y_{1}^{\prime}(1)-3 y_{1}^{\prime}\left(\frac{1}{2}\right)=\mu_{1}, \\
& y_{2}(0)=0, \quad y_{2}^{\prime}(0)=0, \quad 3 y_{2}^{\prime}(1)-4 y_{2}^{\prime}\left(\frac{1}{2}\right)=\mu_{2}, \\
& y_{3}(0)=0, \quad y_{3}^{\prime}(0)=0, \quad 3 y_{3}^{\prime}(1)-2 y_{3}^{\prime}\left(\frac{1}{2}\right)=\mu_{3},
\end{aligned}
$$

where

$$
\begin{aligned}
& f_{1}\left(y_{2}\right)=y_{2}\left(11+1001 e^{-2 y_{2}}\right)\left(11+1101 e^{-4 y_{2}}\right), \\
& f_{2}\left(y_{3}\right)=y_{3}\left(21+1011 e^{-3 y_{1}}\right)\left(21+1111 e^{-5 y_{3}}\right), \\
& f_{3}\left(y_{1}\right)=y_{1}\left(31+1021 e^{-4 y_{1}}\right)\left(31+1121 e^{-6 y_{1}}\right),
\end{aligned}
$$

and

$$
p_{1}(t)=p_{2}(t)=p_{3}(t)=1 .
$$

By direct calculation, we find that

$$
\begin{aligned}
& f_{10}=1125344, \quad f_{20}=1168224, \quad f_{30}=1211904, \\
& f_{1 \infty}=121, \quad f_{2 \infty}=441, \quad f_{3 \infty}=961, \\
& F_{3}=\max \{0.00028178,0.0003932,0.00115891\}=0.00115891, \\
& F_{4}=\min \{0.009015,0.0034013,0.0035676\}=0.0034013 .
\end{aligned}
$$

Applying Theorem 3.1, we get an optimal eigenvalue interval $0.00115891<\lambda_{i}<0.0034013$, $i=1,2,3$ for which boundary value problem (4.3)-(4.4) has at least one positive solution once $\mu_{1}, \mu_{2}$ and $\mu_{3}$ are sufficiently small.

\section{ACKNOWLEDGEMENTS}

The authors thank the referees for their valuable suggestions and comments.

\section{BIBLIOGRAPHY}

1. K. Deimling. Nonlinear functional analysis. Springer-Verlag, New York (1985).

2. J.R. Graef, J. Henderson, B. Yang. Positive solutions to a fourth order three-point boundary value problem // Discr. Contin. Dyn. Syst. 2009(Special), 269-275 (2009).

3. J.R. Graef, B. Yang. Multiple positive solutions to a three-point third order boundary value problem // Discr. Contin. Dyn. Syst. 2005(Special), 337-344 (2005).

4. D. Guo, V. Lakshmikantham. Nonlinear problems in abstract cones. Academic Press, Orlando (1988).

5. J. Henderson, S.K. Ntouyas. Positive solutions for systems of $n^{\text {th }}$ order three-point nonlocal boundary value problems // Electron. J. Qual. Theory Differ. Equ. 2007, 1-12 (2007).

6. J. Henderson, S.K. Ntouyas, I.K. Purnaras. Positive solutions for systems of generalized threepoint nonlinear boundary value problems // Comment. Math. Univ. Carolin. 49:1, 79-91 (2008).

7. J. Henderson, S.K. Ntouyas, I.K. Purnaras. Positive solutions for systems of second order fourpoint nonlinear boundary value problems // Comm. Appl. Anal. 12:1, 29-40 (2008).

8. M.A. Krasnosel'skii. Positive solutions of operator equations. P. Noordhoff Ltd, Groningen (1964).

9. A.G. Lakoud, L. Zenkoufi. Existence of positive solutions for a fourth order three-point boundary value problem // J. Appl. Math. Comput. 50, 139-155 (2016).

10. X. Lin, Z. Zhao. Iterative technique for a third order differential equation with three-point nonlinear boundary value conditions // Electron. J. Qual. Theory Differ. Equ. 2016, 1-10 (2016). 
11. Z. Liu, H. Chen, C. Liu. Positive solutions for singular third order non-homogeneous boundary value problems // J. Appl. Math. Comput. 38, 161-172 (2012).

12. D. Liu, Z. Ouyang. Solvability of third order three-point boundary value problems // Abstr. Appl. Anal. 2014, id 793639 (2014).

13. A.P. Palamides, G. Smyrlis. Positive solutions to a singular third order three-point boundary value problem with an indefinitely signed Green's function // Nonlinear Anal. TMA. 68:7, 2104-2118 (2008).

14. K.R. Prasad, N. Sreedhar, K.R. Kumar. Solvability of iterative systems of three-point boundary value problems // TWMS J. APP. Eng. Math. 3:2, 147-159 (2013).

15. Y. Sun. Positive solutions for third order three-point non-homogeneous boundary value problems // Appl. Math. Lett. 22, 45-51 (2009).

16. Y. Sun, C. Zhu. Existence of positive solutions for singular fourth order three-point boundary value problems // Adv. Difference Equ. 2013:51, 1-13 (2013).

17. C.X. Wang, H.R. Sun. Positive solutions for a class of singular third order three-point nonhomogeneous boundary value problem // Dynam. Syst. Appl. 19, 225-234 (2010).

18. L. Zhao, W. Wang, C. Zhai. Existence and uniqueness of monotone positive solutions for a third order three-point boundary value problem // Differ. Equ. Appl. 10:3, 251-260 (2018).

Kapula Rajendra Prasad

Department of Applied Mathematics,

College of Science and Technology,

Andhra University,

Visakhapatnam, 530 003, India

E-mail: rajendra92@rediffmail.com

Mahanty Rashmita

Department of Applied Mathematics,

College of Science and Technology,

Andhra University,

Visakhapatnam, 530 003, India

E-mail: rashmita.mahanty@gmail.com

Sreedhar Namburi,

Department of Mathematics,

Institute of Science,

GITAM Deemed to be University),

Visakhapatnam, 530 045, India

E-mail: sreedharnamburi13@gmail.com 